\title{
Impact of Optical Micro Ring Resonator as Decoder Switch
}

\author{
Lovepreet Kaur \\ Department of Electronics \& Communication \\ Engineering \\ Shaheed Bhagat Singh State Technical Campus \\ Ferozepur 152004, Punjab, India
}

\author{
Sanjeev Dewra \\ Department of Electronics \& Communication \\ Engineering \\ Shaheed Bhagat Singh State Technical Campus \\ Ferozepur 152004, Punjab, India
}

\begin{abstract}
This paper presents the impact of physical parameters of OMRR like width of ring profile material \& length based on surface Plasmon polaritons using silicon $(\mathrm{Si})$ profile material. It is observed that the received output optical power achieved is $2.99 \mathrm{e}-006 \mathrm{w} / \mathrm{m} 2$ with Silicon $(\mathrm{Si})$ profile material of OMRR at the minimum input transmission power of $0.1 \mathrm{w} / \mathrm{m} 2$ for $1.55 \mu \mathrm{m}$ input transmission wavelength at $0.3 \mu \mathrm{m}$ width of ring. The simulation results in terms of received optical power shows that the silicon $(\mathrm{Si})$ profile material is best profile material for design of OMRR.
\end{abstract}

\section{Keywords}

Optical micro-ring resonator (OMRR), surface plasmon polaritons (SPP), microwave photonic (MWP), Mach-zender interferometer (MZI).

\section{INTRODUCTION}

The main components which are used for applications of optical like optical signal processing telecommunications and biomedical testing and monitoring are integrated optical ring resonators [1-3]. The traditional electronic counterparts limitations are reduced by photonic techniques which have potential applications to distributing, filtering, processing, microwave and radio frequency (RF) signal generating [4-5]. In particularly, the use of integrated photonic circuits and devices to bring down volume and to meet flexible wideband spectral processing requirements, power and weight consumption for both military and civilian applications are of great interest[6]. Because of compact structure and excellent performance, the integrated optical waveguide ring resonators have potential applications for microwave photonics (MWP). Large bandwidth modulators, optical true time delay lines, high sensitivity and phase shifters, and versatile filters have been illustrated recently [7-8]. In earlier years, electro-optic or thermo-optic effects have been demonstrated to control the transmission using ring resonators integrated with phase shifters [9]. In photonic integrated circuits, Micro ring resonators (RRs) are flexible building blocks to realize various optical functionalities such as sensors, filters, modulators, and switches. In its basic configuration, the single ring resonator can be coupled to one or two bus waveguides [10].

Xiao-Yang Zhang et.al [11], demonstrated a tunable MZI coupled optical ring resonator using two alternate analytical models. Analytical expressions are used to explain the desired optimal resonance for arbitrary path length and incident wavelengths by tuning the two integrated phase shifters. In MZI, signals pass through the different sections in the optical. In MZI-FBG based OXC, The MZI is used within two similar FBG's are placed ,two arms of couplers the same is described as a novel architecture of $2 \times 2$ multi wavelength optical cross connects based on optical add/drop multiplexers and optical switches [12-13]. Amr Wageeh et.al [14] proposed a new formula for free spectral range of integrated ring resonators and the estimation of the resonance frequency. The new formula succeeded in explaining the oscillatory variations of the FSR at small ring radius observed in FDTD calculations and also gives much better estimation to the resonance frequency of the ring. It can also be extended with the same technique to better accuracy when considering the second order dispersion in the ring as well as other dispersion types.

Shivam Upadhyay et.al [15] presented a design of 2Dphotonic crystal based pressure sensor and the designed sensor has ring resonator type structure. The photonic sensor is a smart, compact sensing device that can provide facility to use it in magnetic field environment.

Here we introduce the impact of physical parameter like radius of resonator ring on the performance of OMRR in terms of received optical power using Silicon $(\mathrm{Si})$ profile material based on surface plasmon polaritons (SPP) and to simulate and analysis of new optical properties of OMRR the Finite Differences Time domain (FDTD) method.

After the brief introduction, Section 2 presents the system configuration of OMRR, Section 3 presents the results and discussion and Section 4 summarizes the feasibility and conclusion.

\section{SYSTEM COMFIGURATION OF OMRRGE SIZE}

The architecture of hybrid plasmonic optical micro-ring resonator (MRR) based on SPP is designed using Silicon ( $\mathrm{Si}$ ) profile material whose refractive index is 2.1 as shown in Fig.1. The micro-ring resonator (OMRR) contains of unidirectional coupling between an input-output linear waveguides and ring resonator. A basic micro ring resonator contains of a ring waveguides with width (w1) of $0.5 \mu \mathrm{m}, 0.4$ $\mu \mathrm{m} \& 0.3 \mu \mathrm{m}$ as a resonator cavity closely coupled with either one or two linear waveguides as shown in Fig.2. 


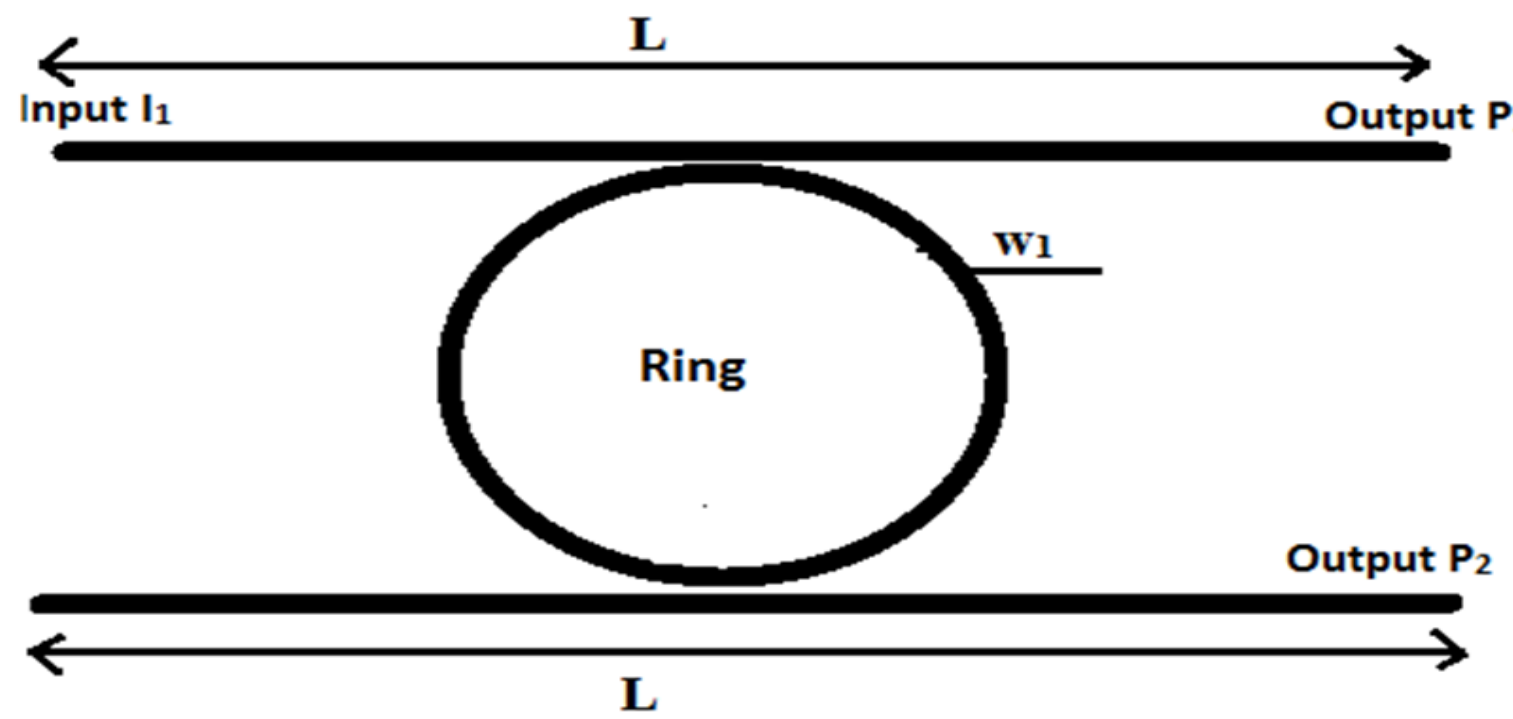

Fig 1: System Configuration of MRR

In this architecture the two linear waveguides are used which has length (L) of $17.4 \mu \mathrm{m}$ and width (w) of $0.5 \mu \mathrm{m}$. For examining the transmission spectra, the light behavior and resonance, we have applied 2D nonlinear finite-difference time-domain (NL-FDTD) method with perfectly matched layer boundary conditions (PML). An optical source transmit the sine-modulated Gaussian pulses signal to the input port of linear waveguide with center wavelength $(\chi)$ of $1.55 \mu \mathrm{m}$, power varies from 0.1 to $0.7 \mathrm{w} / \mathrm{m} 2$, modal index of 2.25 with input field of modal transvers. A part (coupling co-efficient between input waveguide and the ring) of the incoming field is transferred to the ring having radius as shown in the Fig.1. A constructive interference occurred, which is a multiple of the effective wavelength in the optical path length and as a consequence MRR is "On resonance". So, periodic fringes appear at the output ports. At resonance, the drop port shows maximum transmission, since (coupling co-efficient between the ring and output 3 wave guide) of the built up wave inside ring is coupled to this port. A decoder is a circuit which has $2 \mathrm{n}$ outputs and $\mathrm{n}$ inputs, and outputs 1 on the wire according to the binary number represented by the inputs.

The two Z-direction Line optical detectors (OD) are used at the output port (P1) and Output port (P2) of MRR as shown in Fig.1. The optical detectors are applied to measure the normalized optical power at the output port $(\mathrm{P} \neg 1)$ and output port (P2).

\section{RESULTS AND DISCUSSIONS}

This paper presents the architecture of OMRR based on SPP using Silicon $(\mathrm{Si})$ profile materials whose refractive index of 2.1 using 2-D FDTD simulation method for analyzing the results in terms of received optical power. When light reaches a steady state and calculated by using the two Z-direction optical detectors at the output port of OMRR more focus is given to the power of each port. The normalized power at port 1 and port 2 as a function of materials which is used for designing the OMRR for $1550 \mathrm{~nm}$ input wavelength. When the proprieties of micro-ring resonator region are changed like Length, width \& materials due to this the normalized power at output port of ODC is also varied. Two Z-direction line optical power detectors at the output port of ODC are used to find the output normalized power. The variations of received normalized optical power at port1 and port 2 are dependent upon the micro-ring resonator region proprieties like length of region, width and material profile of OMRR.

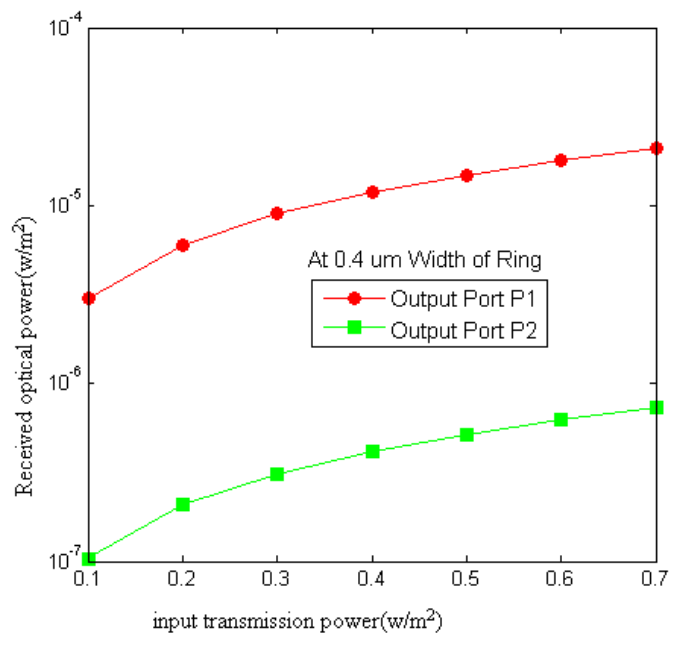

Figure.2. received optical power versus input transmission power at $0.4 \mu \mathrm{m}$ width of Ring

Fig.2. depicts the received optical power versus input transmission power at $1550 \mathrm{~nm}$ input transmission wavelength by using Silicon profile material at $0.4 \mu \mathrm{m}$ width of ring for OMRR. It is observed from the plot when the input transmission power is changed from 0.1 to $0.8 \mathrm{w} / \mathrm{m} 2$ then the received optical powers are also increased from $2.99 \mathrm{e}-006$ to $2.09 \mathrm{e}-005$ and $1.03 \mathrm{e}-007$ to $7.27 \mathrm{e}-007$ at both output port (P1, $\mathrm{P} 2$ ) respectively. The variation of received optical power at the output $\mathrm{p} 1$ and $\mathrm{p} 2$ are depend upon the properties like length \& width of micro-ring resonator and material profile. 


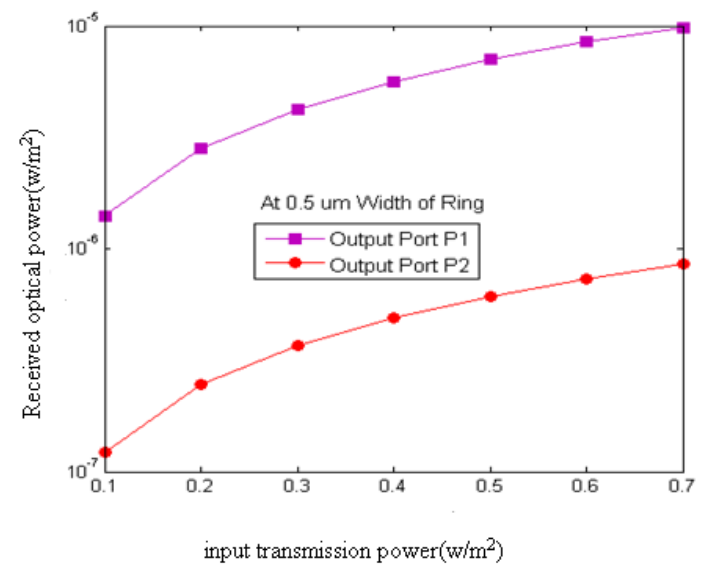

Figure3. received optical power versus input transmission power at $0.5 \mu \mathrm{m}$ width of Ring

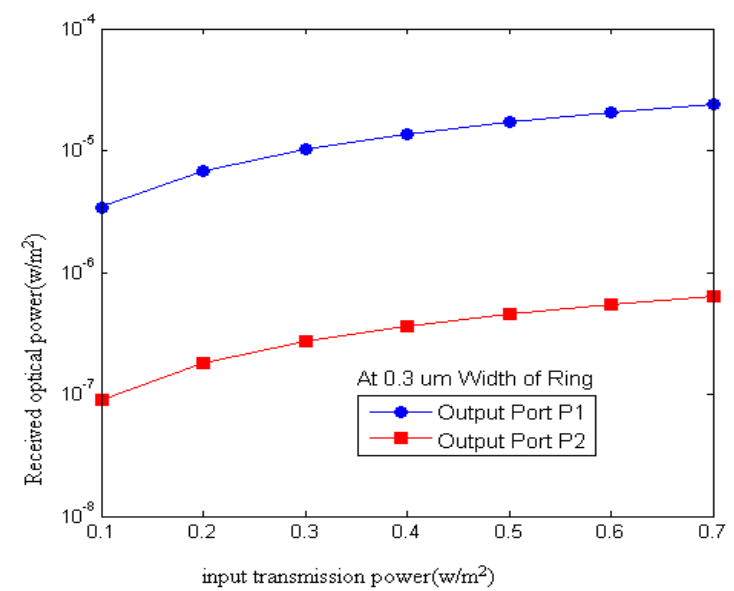

Figure.4. received optical power versus input transmission power at $0.3 \mu \mathrm{m}$ width of Ring

Fig.3. Shows the output received optical power versus input transmission power at $1550 \mathrm{~nm}$ input transmission wavelength by using $\mathrm{Si}$ (Silicon) material profile for OMRR at $0.5 \mu \mathrm{m}$ width of ring. When the input Transmission power is varies from 0.1 to $0.8 \mathrm{w} / \mathrm{m} 2$ then the output received optical power is also increased from 1.410e-006 to9.87e-006 and 1.226e-007 to $8.58 \mathrm{e}-007$ at port $\mathrm{p} 1$ and $\mathrm{p} 2$ respectively. This graph indicates that the variation of received optical at $\mathrm{p} 1$ and $\mathrm{p} 2$ are depend upon the OMRR properties.

The graph of received optical power versus input transmission power at $1550 \mathrm{~nm}$ input transmission wavelength by using Silicon $(\mathrm{Si})$ material profile for OMRR at $0.3 \mu \mathrm{m}$ width of ring is shown in Figure 4. It is shown from the graph that the received optical power increases from $3.43 \mathrm{e}-006$ to $2.40 \mathrm{e}-005$ at output port( p1 ) and from $9.09 \mathrm{e}-008$ to $6.365 \mathrm{e}-007$ at output port ( p2) when the input transmission power deviates from 0.1 to $0.8 \mathrm{w} / \mathrm{m} 2$. At both output port, the variation in the received optical power is a function of OMRR parameters.

\section{CONCULSION}

The design of OMRR based on SPP using FDTD simulation method is analyzed using profile material Silicon $(\mathrm{Si})$. The analysis shows better results in terms of received optical power using silicon profile material for designing the OMRR. It is observed that the maximum received optical power is achieved in Silicon $(\mathrm{Si})$ profile material of OMRR at the minimum input transmission power for $1.55 \mu \mathrm{m}$ input transmission wavelength at $0.3 \mu \mathrm{m}$ width of ring. Optical filters, resonators, waveguides, multiplexers, logical gates, optical decoder switches etc can be designed by using the photonic crystal.

\section{ACKNOWLEDGMENTS}

Lovepreet kaur was born in Punjab, India she graduated as Electronic and comm. Engineering (2015) from Punjab Technical University. She is currently doing M.Tech Electronic and comm. engineering under Maharaja University of Punjab.3. Tables, Figures and Equations

\section{REFERENCES}

[1] Q. Xu, D. Fattal, and R. G. Beausoleil, "Silicon micro ring resonators with 1.5 - $\mu \mathrm{m}$ radius," Opt. Express, vol. 16, no. 6, pp. 4309-4315,Mar. 2008.

[2] Q. Xu, B. Schmidt, S. Pradhan, and M. Lipson, "Micrometre-scale silicon electro-optic modulator," Nature, vol. 435, pp. 325-327, May 2005.

[3] T. Mamdouh and D. Khalil, "A MEMS tunable optical ring resonator filter," J. Opt. Quantum Electron., vol. 37, no. 9, pp. 835-853, Jul. 2005.

[4] Robert A. Minasian, "Photonic signal processing of microwave signals",IEEE Trans. on Micro. Theo. and Tech., 54(2): 832-84, 2006.

[5] J. Capmany, J. Mora, I. Gasulla, et al., "Microwave photonic signal processing", J. Lightw. Technol., 31(4): 571-586, 2013.

[6] D. A. I. Marpaung, C. G. H. Roeloffzen, R. G. Heideman, et al., "Integrated microwave photonics", Laser Photonics Rev., DOI:10.1002/Ipor.201200032, 2013.

[7] M. Burla, D. Marpaung, L. Zhuang, et al., "On-chip CMOS compatible reconfigurable optical delay line with separate carrier tuning for microwave photonic signal processing”, Opt. Express, 19(22): 21475-21484, 2011.

[8] J. Lloret, J. Sancho, M. Pu, et al., "Tunable complexvalued multi-tap microwave photonic filter based on single silicon-on-insulator microring resonator", Opt. Express, 19(13): 12402-12407, 2011.

[9] Xiao-Yang Zhang, Tong Zhang, et.al, "Tunable Optical Ring Resonator Integrated With Asymmetric MachZehnder Interferometer", Journal of Lightwave Technology, vol. 28, no. 17, September 1, 2010.

[10] S. Darmawan, Y. M. Landobasa, et.al,"Nested-Ring Mach-Zehnder Interferometer in Silicon-on-Insulator", IEEE Photonics Technology Letters, Vol. 20, No. 1, January 1, 2008.

[11] Sanjeev Dewra, R.S. Kaler, "Performance analysis of optical network based on optical add drop multiplexers with different MZI techniques" Optik, 124 (2013) 347351

[12] Sanjeev Dewra, R.S. Kaler, “ Crosstalk analysis in an optical network based on optical cross connects with different MZI techniques" Optik 124 (2013) 55- 59

[13] Amr Wageeh, Salwa El-Sabban and Diaa Khalil, "Resonance Wavelength of Integrated Optical Ring Resonator With Small Radius of Curvature" IEEE Photonics Technology Letters, vol. 26, no. 7, pp.641644,April 2014.

[14] Shivam Upadhyay and vijay Laxmi Kalyani "Designing of Ring -Resonator based Photonic Pressure Sensor" Imperial Journal of Interdisciplinary Research (IJIR) Vol-2, Issue-4, 2016. 